\title{
Measurement of polarization dependent loss, polarization mode dispersion and group delay of optical components using swept optical single sideband modulated signals
}

\author{
Mikel Sagues, Miguel Pérez and Alayn Loayssa \\ Departamento de Ingeniería Eléctrica y Electrónica, Universidad Pública de Navarra \\ Campus Arrosadia s/n, 31006 Pamplona (Spain) \\ alayn.loayssa@unavarra.es \\ http://www.iee.unavarra.es
}

\begin{abstract}
We introduce a novel method for high wavelength-resolution measurement of polarization-averaged group delay, polarization dependent loss and polarization mode dispersion of optical components using swept optical single sideband modulated signals. Measurements of a phase-shifted fiber Bragg grating, a chirped fiber Bragg grating and of the Brillouin spectra of a length of fiber are used in order to demonstrate the technique.

(C)2008 Optical Society of America

OCIS codes: (999.9999) Single sideband modulation; (290.5900) Scattering, stimulated Brillouin; (070.1170) Analog optical signal processing; (999.9999) Microwave photonics.
\end{abstract}

\section{References and links}

1. J. E. Román, M. Y. Frankel, and R. D. Esman, "Spectral characterization of fiber gratings with high resolution,” Opt. Lett. 23, 939-941 (1998).

2. R. Hernández, A. Loayssa, and D. Benito, "Optical vector network analysis based on single-sideband modulation," Opt. Eng. 43, 2418-2421 (2004).

3. A. Loayssa, R. Hernández, D. Benito, and S. Galech, "Characterization of stimulated Brillouin scattering spectra by use of optical single-sideband modulation," Opt. Lett. 29, 638-640 (2004).

4. D. J. Krause, J.C. Cartledge, L. Jakober, and K. Roberts, "Measurement of passive optical components using a carrier and single sideband," in Proc. Optical Fiber Communications Conference, (OFC'2006) paper OFN5 (2006).

5. T. Kawanishi, T. Sakamoto, M. Izutsu, D. Fonseca, A. Cartaxo, and P. Monteiro, "Fine transmittance/reflectivity measurement system using single-sideband frequency sweeper with ultra-wideband Hilbert transformer," in Proc. European Conference on Optical Communication (ECOC'2006) paper Mo.4.4.6 (2006).

6. M. Sagues, G. Beloki, and A. Loayssa, "Broadband Swept Optical Single-sideband Modulation Generation for Spectral Characterization of Optical Components," in Proc. European Conference on Optical Communication (ECOC'2007) paper 6.6.6 (2007).

7. T. Niemi, M. Uusimaa, and H. Ludvigsen, "Limitations of phase-shift method in measuring dense group delay ripple of fiber Bragg gratings," IEEE Photon. Technol. Lett. 13, 1334-1336 (2001).

8. G. D. VanWiggeren, A. R. Motamedi, and D. M. Barley, "Single-Scan Interferometric Component Analyzer," IEEE Photon. Technol. Lett. 15, 263-265 (2003).

9. D. K. Gifford, B. J. Soller, M. S. Wolfe, and M. E. Froggatt, "Optical vector network analyzer for single-scan measurements of loss, group delay, and polarization mode dispersion," Appl. Opt. 44, 7282-7286 (2005).

10. B. L. Heffner, "Deterministic, analytically complete measurement of polarization dependent transmission through optical devices," IEEE Photon. Technol. Lett. 4, 1066-1069 (1992).

11. B. L. Heffner, "Automated Measurement of Polarization Mode Dispersion Using Jones Matrix Eigenanalysis," IEEE Photon. Technol. Lett. 4, 451-454 (1992).

12. R. M. Craig, "Accurate Spectral Characterization of Polarization-Dependent Loss," J. Lightwave Technol. 21, 432-437 (2003).

13. P. A. Williams, "Modulation phase-shift measurement of PMD using only four launched polarization states: a new algorithm," Electron. Lett. 35, 1578-1579 (1999). 
14. Y. Shi, L. Yan, and X. S. Yao, "Automatic Maximum-Minimum Search Method for Accurate PDL and DOP Characterization,” J. Lightwave Technol. 24, 4006-4012 (2006).

15. O. H. Adamczyk, A. B. Sahin, Q. Yu, S. Lee, and A. E. Willner, "Statistics of PMD-induced power fading for intensity-modulated double-sideband and single-sideband microwave and millimeter-wave signals," IEEE Trans. Microwave Theory Tech. 49, 1962-1967 (2001).

16. M. Oskar van Deventer and A. J. Boot, "Polarization properties of stimulated Brillouin scattering in singlemode fibers," J. Lightwave Technol. 12, 585-590 (1994).

\section{Introduction}

The precise characterization of the response of optical devices is of paramount importance in order to develop links and networks that push the limits of the information carrying capacity of optical fibers. There are commercial instruments available for this task. However, there is still room for enhancement in a number of specifications of measurement systems. One of them is wavelength-resolution. Recently, the use of optical single sideband (OSSB) modulation has been proposed to provide extremely high wavelength-resolution spectral characterization of optical components and systems [1-6]. This is an alternative to traditional modulation phase-shift [7] and interferometric methods [8] that can achieve resolutions in the $\mathrm{Hz}$ range.

To date the OSSB technique could only be applied to the measurement of polarization-independent components. However, full polarization-resolved measurements of parameters such as polarization dependence loss (PDL), polarization mode dispersion (PMD) and polarization-averaged group delay (PAGD) are necessary for the characterization of real-world optical components. A number of techniques have been proposed for the measurement of those parameters [9-14], but none of them yields the spectral resolution that could be achieved with OSSB characterization. Moreover, existing approaches to extend the capabilities of spectral characterization methods to polarization measurements, such as the Mueller-matrix [12] or the 'four-points' [13] methods, are not applicable to the OSSB technique.

In this paper, we introduce the theoretical fundamentals and the experimental implementation of the method that we have devised to provide polarization measurement capability to the swept OSSB technique.

\section{Theory}

The use of OSSB signals for spectral characterization of polarization-independent components is based on the correspondence or mapping that this modulation format provides between the optical and the electrical domains [1]. The electric field of an OSSB signal at the input of a device under test (DUT) can be expressed as:

$$
E_{\text {in }}(t)=A_{O C} \exp \left(j 2 \pi v_{0} t\right)+A_{S B} \exp \left[j 2 \pi\left(v_{0}+f_{R F}\right) t\right]
$$

where $v_{0}$ and $v_{0}+f_{R F}$ are the frequency of the optical carrier and sideband, respectively, and $A_{O C}$ and $A_{S B}$ are their complex amplitudes. In the technique, this signal is propagated though the DUT, which has an optical transfer function $H(v)$, and is finally detected in a wideband photodetector (PD). Analyzing the detected RF we find that the electrical current at $f_{R F}$ is proportional to [2]:

$$
\begin{aligned}
\left.i_{\text {out }}(t)\right|_{f=f_{R F}} & \propto\left|A_{O C}\left\|A_{S B}\right\| H\left(v_{0}\right) \| H\left(v_{0}+f_{R F}\right)\right| \times \\
& \times \cos \left[2 \pi f_{R F} t+\operatorname{Arg} A_{S B}-\operatorname{Arg} A_{O C}+\operatorname{ArgH}\left(v_{0}+f_{R F}\right)-\operatorname{ArgH}\left(v_{0}\right)\right]
\end{aligned}
$$

If the contribution of the measurement system is calibrated out this simplifies to:

$$
I\left(f_{R F}\right)=H^{*}\left(v_{0}\right) \cdot H\left(v_{0}+f_{R F}\right)
$$

\#98545 - \$15.00 USD

(C) 2008 OSA 


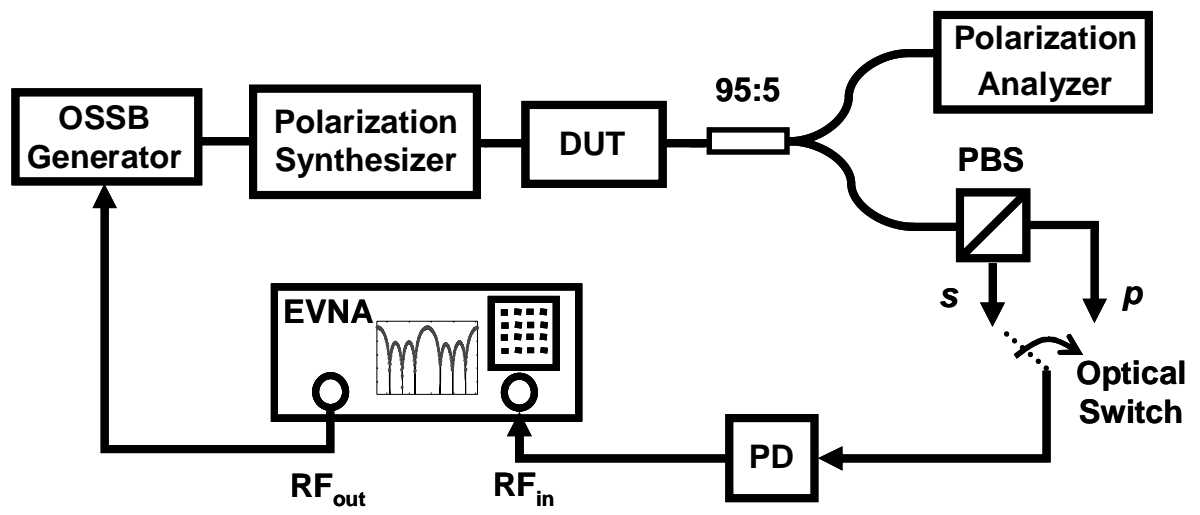

Fig. 1. OSSB Measurement system setup.

where phasorial notation is deployed. Notice that from the amplitude and phase-shift of the detected electrical signal it is possible to extract the optical transfer function of the DUT by sweeping the modulation frequency $f_{R F}$ while $v_{0}$ is kept constant [2].

However, (2) is only valid under the assumption that the state of polarization (SOP) of carrier and sideband at the output of the DUT are identical, which is not valid in general for polarization-dependent devices. If the optical carrier and the sideband at the output of the DUT have different SOP the detected photocurrent will depend not only on the phase-shift and amplitude transfer function of the DUT at the wavelengths of carrier and sideband, but also on the difference in SOP between them. For instance, it has been shown that propagation of an OSSB signal through a medium with DGD leads to an RF signal fading that in this measurement technique would introduce errors in the amplitude measurement [15].

In order to bypass this limitation we propose a technique which is based on sequentially generating two OSSB sweeps with orthogonal linear SOP and, for each one, analyzing two orthogonal polarizations at the output of the DUT using a polarization beam splitter (PBS). Fig. 1 depicts the fundamentals of the proposed measurement setup. An OSSB signal is generated by an Electrical Vector Network Analyzer (EVNA) and fed to a polarization synthesizer whose output optical signal $\vec{E}_{i n}$ can be expressed in Jones Vector form as:

$$
\vec{E}_{i n}=\left(\begin{array}{l}
\left|A_{O C}\right| \cdot\left|E_{S}\right| \cdot e^{j \cdot \delta_{S}^{O C}}+\left|A_{S B}\right| \cdot\left|E_{S}\right| \cdot e^{j \cdot \delta_{S}^{S B}} \cdot e^{j \cdot 2 \pi \cdot f_{R F} \cdot t} \\
\left|A_{O C}\right| \cdot\left|E_{p}\right| \cdot e^{j \cdot \delta_{p}^{O C}}+\left|A_{S B}\right| \cdot\left|E_{p}\right| \cdot e^{j \cdot \delta_{p}^{S B}} \cdot e^{j \cdot 2 \pi \cdot f_{R F} \cdot t}
\end{array}\right) \cdot e^{j \cdot 2 \pi \cdot v_{o} \cdot t}
$$

where the sideband and the optical carrier have SOP, which are given by $\left|E_{s, p}\right|$ and $\delta_{s, p}^{O C, S B}$, the amplitudes and optical phase-shifts associated with the two orthogonal polarization axes. For simplicity, we choose the polarization axes $s$ and $p$ defined by the PBS as reference coordinate system.

The signal from the synthesizer goes through the DUT and at the output its orthogonal polarization components are separated at the PBS. Finally, an optical switch is used to select either of the ports of the PBS and detect its output signal in a wideband photodetector (PD). Alternatively, two PDs could be attached to each port of the PBS so as to double the measurement speed. The electric fields at the outputs of the PBS are given by:

$$
\begin{aligned}
& \vec{E}_{s}=\bar{H}_{s} \cdot \bar{H}_{D U T} \cdot \vec{E}_{i n} \\
& \vec{E}_{p}=\bar{H}_{p} \cdot \bar{H}_{D U T} \cdot \vec{E}_{i n}
\end{aligned}
$$

\#98545 - \$15.00 USD

(C) 2008 OSA 
where $\bar{H}_{D U T}, \bar{H}_{s}$ and $\bar{H}_{p}$ are the $2 \times 2$ complex-coefficient matrices describing the frequency dependent optical transfer functions of the DUT and of the output ports $s$ and $p$ of the PBS, i.e. their wavelength-dependent Jones matrices. The detected current will be proportional to the inner product of the received electric field [10]. This electrical signal is applied to the input port of the EVNA, where the measured component at $f_{R F}$ is given by:

$$
\begin{aligned}
I_{s}\left(f_{R F}\right) \propto & \left|A_{O C}\right| \cdot\left|A_{S B}\right| \cdot\left[H_{11}^{*}\left(v_{0}\right) \cdot H_{11}\left(v_{0}+f_{R F}\right) \cdot\left|E_{s}\right|^{2} \cdot e^{j\left(\delta_{s}^{S B}-\delta_{s}^{O C}\right)}+\right. \\
& H_{11}^{*}\left(v_{0}\right) \cdot H_{12}\left(v_{0}+f_{R F}\right) \cdot\left|E_{s}\right| \cdot\left|E_{p}\right| \cdot e^{j\left(\delta_{p}^{S B}-\delta_{s}^{O C}\right)}+ \\
& H_{12}^{*}\left(v_{0}\right) \cdot H_{11}\left(v_{0}+f_{R F}\right) \cdot\left|E_{s}\right| \cdot\left|E_{p}\right| \cdot e^{j\left(\delta_{s}^{S B}-\delta_{p}^{O C}\right)}+ \\
& \left.H_{12}^{*}\left(v_{0}\right) \cdot H_{12}\left(v_{0}+f_{R F}\right) \cdot\left|E_{p}\right|^{2} \cdot e^{j\left(\delta_{p}^{S B}-\delta_{p}^{O C}\right)}\right] \cdot e^{j 2 \pi \cdot \tau_{s} \cdot f_{R F}} \\
I_{p}\left(f_{R F}\right) \propto & \left|A_{O C}\right| \cdot\left|A_{S B}\right| \cdot\left[H_{21}^{*}\left(v_{0}\right) \cdot H_{21}\left(v_{0}+f_{R F}\right) \cdot\left|E_{s}\right|^{2} \cdot e^{j\left(\delta_{s}^{S B}-\delta_{s}^{O C}\right)}+\right. \\
& H_{21}^{*}\left(v_{0}\right) \cdot H_{22}\left(v_{0}+f_{R F}\right) \cdot\left|E_{s}\right| \cdot\left|E_{p}\right| \cdot e^{j\left(\delta_{p}^{S B}-\delta_{s}^{O C}\right)}+ \\
& H_{22}^{*}\left(v_{0}\right) \cdot H_{21}\left(v_{0}+f_{R F}\right) \cdot\left|E_{s}\right| \cdot\left|E_{p}\right| \cdot e^{j\left(\delta_{s}^{S B}-\delta_{p}^{O C}\right)}+ \\
& \left.H_{22}^{*}\left(v_{0}\right) \cdot H_{22}\left(v_{0}+f_{R F}\right) \cdot\left|E_{p}\right|^{2} \cdot e^{j\left(\delta_{p}^{S B}-\delta_{p}^{O C}\right)}\right] \cdot e^{j 2 \pi \cdot \tau_{p} \cdot f_{R F}}
\end{aligned}
$$

where $I_{s, p}$ are the phasorial currents at $f_{R F}$ for the two ( $s$ and $p$ ) output ports of the PBS, $\tau_{s, p}$ are the optical delays associated to each output port and $H_{i j}(v)$ the complex coefficients of the transfer function matrix of the DUT (where $i$ determines the row and $j$ the column). From this expression we can obtain the detected RF amplitude and phase-shift for each orthogonal polarization OSSB sweep at the input. After a calibration process in which the DUT is removed and the frequency response of the system is measured at the two outputs of the PBS for the two orthogonal input states of polarization, (7) simplifies to the following expressions relating the measured photocurrent and the complex coefficients of the transfer function of the DUT:

$$
\begin{aligned}
& I_{s}^{L H}\left(f_{R F}\right)=H_{11}^{*}\left(v_{0}\right) \cdot H_{11}\left(v_{0}+f_{R F}\right) \\
& I_{s}^{L V}\left(f_{R F}\right)=H_{12}^{*}\left(v_{0}\right) \cdot H_{12}\left(v_{0}+f_{R F}\right) \\
& I_{p}^{L H}\left(f_{R F}\right)=H_{21}^{*}\left(v_{0}\right) \cdot H_{21}\left(v_{0}+f_{R F}\right) \\
& I_{p}^{L V}\left(f_{R F}\right)=H_{22}^{*}\left(v_{0}\right) \cdot H_{22}\left(v_{0}+f_{R F}\right)
\end{aligned}
$$

where $I_{s, p}^{L H, L V}$ are the measured phasorial currents at the two ports of the PBS ( $s$ and $p$ ) for the two linear-horizontal (LH) or linear-vertical (LV) polarization input sweeps. From these expressions, the wavelength-dependent Jones Matrices of the DUT are derived and then used to calculate PAGD, PDL and PMD as a function of wavelength. Notice that this solution requires the previous determination of the Jones Matrix for the optical carrier. However, this 
can be easily done just before the sweep measurement using the standard procedure [10], which provides the matrix transfer function of the DUT at the optical carrier within a complex unknown constant $C$, i.e., $\tilde{H}\left(v_{0}\right)=C \cdot \bar{H}\left(v_{0}\right)$, where $\tilde{H}\left(v_{0}\right)$ is the measured Jones Matrix and $\bar{H}\left(v_{0}\right)$ the true matrix transfer function.

For the calculation of the PAGD we use the definition given in [9]:

$$
\operatorname{PAGD}(v)=\frac{\operatorname{Arg}\left\{\sum H_{i j}(v) \cdot H_{i j}{ }^{*}(v-\Delta v)\right\}}{2 \pi \Delta v}=\frac{\operatorname{Arg}\left\{\frac{1}{|C|^{2}} \cdot \sum \tilde{H}_{i j}(v) \cdot \tilde{H}_{i j}{ }^{*}(v-\Delta v)\right\}}{2 \pi \Delta v}
$$

where $\Delta v$ is the optical frequency separation between measured wavelengths. It can be seen that $|C|^{2}$ can be removed from the equation.

The maxima and minima of the optical transmission of the DUT are given by the eigenvalues of the matrix $\bar{H}(v)^{* T} \cdot \bar{H}(v)$ [10]. Therefore, for a given wavelength the measured maximum and minimum optical intensity transmission are given by:

$$
T_{\max } \text { and } T_{\min }=\frac{1}{|C|^{2}} \cdot \text { Eigenvalues }\left[\bar{H}(v)^{* T} \cdot \bar{H}(v)\right]
$$

PDL is defined as the ratio between the maximum and minimum transmission ( $P D L=T_{\max } / T_{\min }$ ), so that the constant $C$ in (10) will not affect the measurement of this parameter.

DGD is given by [11]:

$$
D G D(v)=\left|\frac{\operatorname{Arg}\left(\rho_{1}(v) / \rho_{2}(v)\right)}{2 \pi \cdot \Delta v}\right|
$$

where $\rho_{1}(v)$ and $\rho_{2}(v)$ are the eigenvalues of the matrix $\bar{H}(v+\Delta v) \cdot \bar{H}^{-1}(v)$. It can be shown that $\rho_{1}(v)$ and $\rho_{2}(v)$ can be determined within a complex constant by the calculation of the eigenvalues of $\tilde{H}(v+\Delta v) \cdot \tilde{H}^{-1}(v)$ so that the ratio $\rho_{1}(v) / \rho_{2}(v)$ is also independent of C.

\section{Experimental setup and measurements}

In order to proof the feasibility of this measurement system we have performed several example measurements. The experimental setup is the one described in Fig. 1. The 95:5 coupler in the setup sends a sample of the output signal from the DUT to a polarization analyzer that is used for the calibration of the polarization axes as well as for the measurement of the Jones matrix of the optical carrier. The coupler and the pigtails are non-polarizing components that simply introduce a rotation on the SOP, but have negligible effect on the measured PDL, DGD or PAGD [9].

The OSSB generator was implemented using an external cavity laser (Santec, TSL-210) modulated by a dual-drive Mach-Zehnder modulator and a $90^{\circ}$-hybrid coupler driven by a $10 \mathrm{dBm}$ RF signal [1]. However, other methods that provide higher quality OSSB modulation with optical carrier could be used as well [6]. The deployed laser source has a certified linewidth of $<500 \mathrm{KHz}$ and a frequency stability of $<3 \mathrm{pm}$ for 30 minutes. In addition, the frequency stability of the laser source was monitored during the measurements by the use of a wavelength meter with $1 \mathrm{pm}$ accuracy. Fig. 2 shows the OSSB spectrum at the output of the modulator. The modulator was set so that a $20 \mathrm{~dB}$ unwanted sideband suppression (defined as the ratio in $\mathrm{dB}$ between the two main sidebands of the signal) was achieved in a $17 \mathrm{GHz}$ frequency range from $1 \mathrm{GHz}$ to $18 \mathrm{GHz}$, limited by the available hybrid coupler [1]. A 
photodetector with $50 \mathrm{GHz}$ bandwidth and a responsivity of $0.65 \mathrm{~A} / \mathrm{W}$ is used. Finally an EDFA providing $20 \mathrm{~dB}$ of optical gain and a low noise RF amplifier $(25 \mathrm{~dB})$ were deployed at the output of the OSSB generator and the PD, respectively, in order to increase the dynamic range of the measurement.

In the method that we propose, expression (8) is used to derive the matrix transfer function of the DUT. However, this derivation is just possible if all the coefficients of the Jones matrix at the carrier wavelength are not null. In our setup, this is ensured by introducing an automatic polarization rotation so as to avoid that the SOP at the input of the PBS matches any of the principal axis of the PBS. It can be shown that this rotation has no effect on the measurements [9].

Figure 3 shows the characterization of the PDL of a commercial PS-FBG from AOS (Advanced Optical Systems) and Fig. 4 shows the measurement of the DGD of a C-FBG. In both measurements a $17-\mathrm{GHz}$ optical frequency sweep was performed with $10-\mathrm{MHz}$ resolution and a sweep time of $13.57 \mathrm{~s}$. Measurements using the OSSB method (red solid line) are compared to those using the modulation phase-shift method (symbols) with 2-pm resolution and $125-\mathrm{MHz}$ frequency modulation [12-13]. Both measurements show good agreement. The small deviations are attributed to the lower resolution of the modulation phase-shift method, the residual PDL and PMD of the components in our current setup and also to the degree of sideband suppression ratio that we had in our OSSB modulation [2].

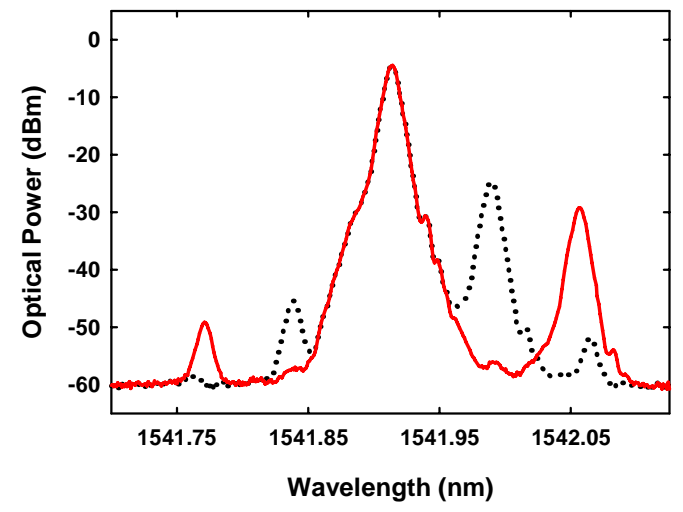

Fig. 2. Spectrum of the OSSB modulated signal at the modulator output for $9.5 \mathrm{GHz}$ (black dotted line) and $18 \mathrm{GHz}$ (red line) modulation frequency.

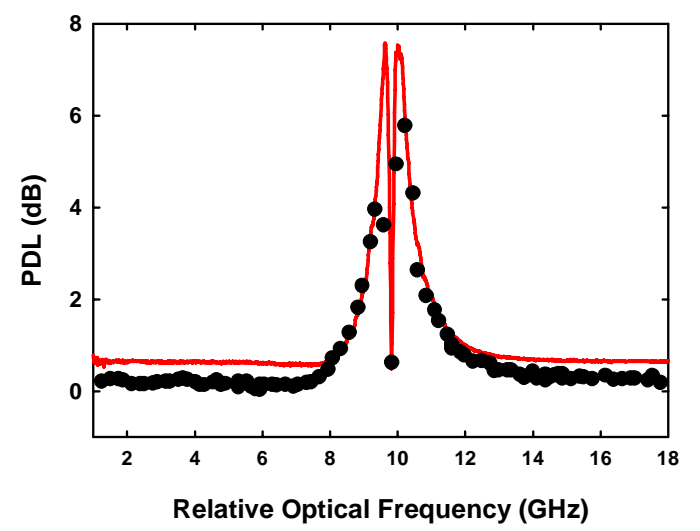

Fig. 3. Measurement of PDL of a PS-FBG using the OSSB technique (red solid line) and modulation phase-shift method (symbols).

\#98545 - \$15.00 USD

(C) 2008 OSA 


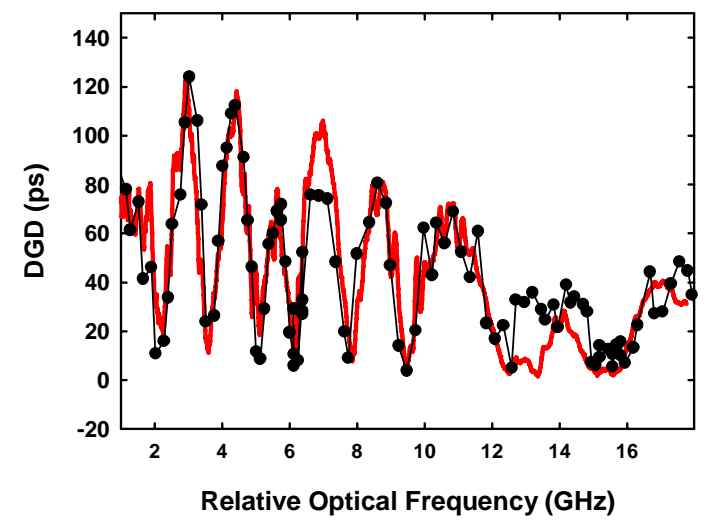

Fig. 4. Measurement of DGD of a C-FBG using the OSSB technique (red solid line) and modulation phase-shift method (thin line with symbols).
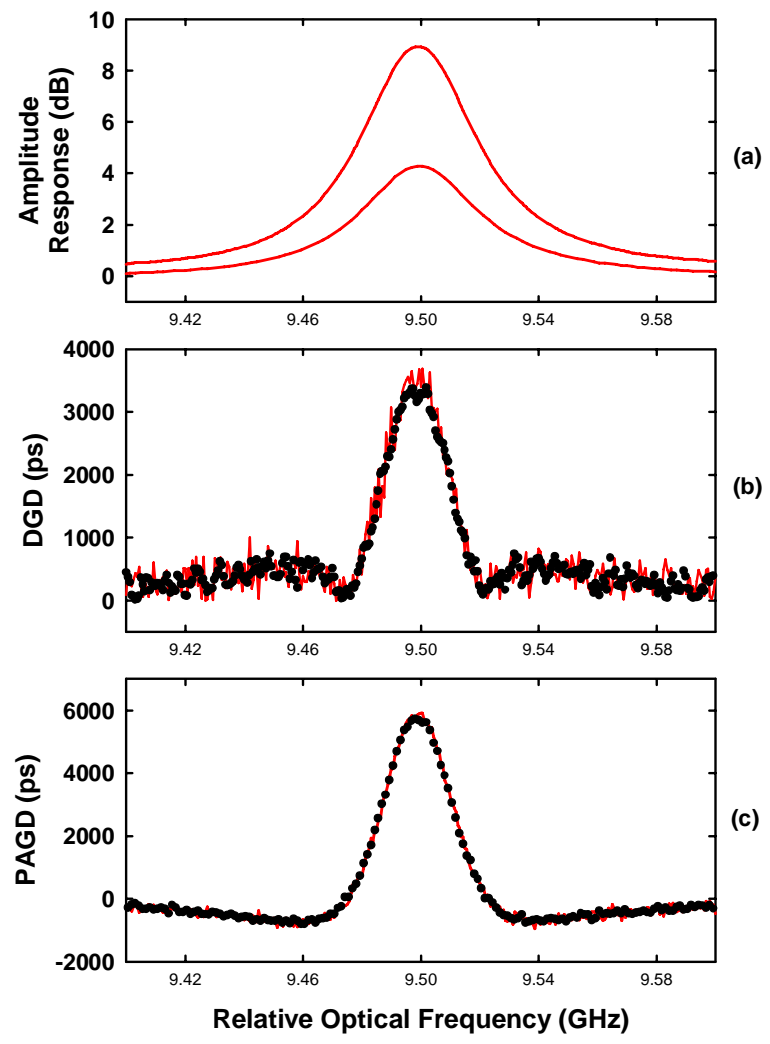

Fig. 5. Measurement of (a) PDL (maximum and minimum amplitude response are shown), (b) DGD and (c) PAGD of the Brillouin spectra of a length of dispersion compensating fiber. Measurements (red line) are compared to theoretical calculations (black symbols) obtained by applying Kramers-Kronig equations to the measured amplitude response.

\#98545 - \$15.00 USD

(C) 2008 OSA 
Finally, in order to show the high-resolution capabilities of the technique we have performed the characterization of the stimulated Brillouin scattering (SBS) spectra of a 2-Km length of dispersion compensating fiber. Fig. 5 highlights the PDL, DGD and PAGD that was measured when an amplified sample of the optical carrier was counterpropagated in the fiber so as to excite Brillouin gain on the OSSB sideband [3]. The resolution of the measurement was $625 \mathrm{KHz}$ with a sweep time of $15.46 \mathrm{~s}$. Note that, as it is predicted by theory [16], a low-birefringence fiber with SBS interaction behaves as a medium with wavelength-dependent diattenuation. Moreover, Kramers-Kronig relationships imply that it is also a medium with wavelength-dependent birefringence. The narrow bandwidth of SBS spectra does not allow its accurate characterization using modulation phase-shift method. Therefore, in order to independently verify our characterization DGD and PAGD measurements in Fig. 5 (red line) are compared to theoretical calculations (black symbols) obtained by applying Kramers-Kronig equations to the measured amplitude response. It can be seen that the agreement is excellent.

\section{Conclusions}

In conclusion, we have introduced a method to measure polarization-related parameters of optical components using swept OSSB modulated signals. This brings full measurement capabilities to this technique for the characterization of optical components, overcoming the classical limitation of OSSB characterization technique [1-2]. A mathematical derivation has been presented and experimental results have been shown in order to demonstrate the technique. Future research should focus on applying this technique to OSSB generation methods with higher sideband suppression [6].

\section{Acknowledgments}

The authors wish to acknowledge financial support from the Spanish Ministerio de Educación y Ciencia through the project TEC2007-67987-C02-02, and from the Gobierno de Navarra through the research project 13.326 .

\#98545 - \$15.00 USD

(C) 2008 OSA 